\title{
Assessing Knowledge, Attitude, and Practices on Hospital Acquired Infections Control by Anaesthesia Practitioners in the Operating Theatres of the Tamale Teaching Hospital (TTH); Ghana
}

\author{
Martin Mumuni Danaah Malick ${ }^{1}$, Edem Yao Akpa ${ }^{2}$, Peter Paul Bamaalabong ${ }^{3}$, \\ ${ }^{1}$ University for Development Studies, Department of Pharmacy, \\ ${ }^{2}$ University for Development Studies, Department of Anesthesiology and Intensive Care, \\ ${ }^{3}$ University for Development Studies, Department of Anesthesiology and Intensive Care, \\ *Corresponding Author: Martin Mumuni Danaah Malick; mdanaah@uds.edu.gh
}

Received 03 May 2020;

Accepted 30 May 2020;

Published 13 June 2020

\begin{abstract}
Background: Hospital Acquired Infections (HAIs) place a significant economic burden on the healthcare system. Infection control practices are important in minimizing healthcare associated infections. However, low compliance with Universal and Standard Precautions has been reported in a number of studies. The Centre for Disease Control and Prevention (CDC) developed baseline definitions for HAIs that were republished in 2004 and has defined HAIs as those that develop during hospitalization but are neither present nor incubating upon the patient's admission to the hospital; generally, these infections occur between 48 to 72 hours after admission and within 10 days after hospital discharge. this study aimed at unveiling the level of knowledge, attitude and practices on infection prevention control in the operating theatres by anaesthesia practitioners at TTH. Materials and Methods: A cross-sectional study design was employed. A mixed-method approach was used for data collection which includes a structured questionnaire carried out via face to face interview and observation. Results: The study showed that $100 \%$ of the respondents have knowledge on hospital acquired infection control in the theatre in one way or the other whereas attitude and practices toward hospital infection control in the operating theatres are undesirable in some specific areas of infection control such as wearing of sterile gowns and goggle. As high as $80.6 \%$ and $69.4 \%$ do not wear goggle and gowns respectively whilst performing regional anaesthesia. Conclusions: This study demonstrated that anaesthetists at TTH have reported sub-optimal levels of compliance i.e. attitude and practices with selective infection control. The study further demonstrated that discrepancies exist between anaesthetists' attitudes towards a guideline as well as their actual practice.
\end{abstract}

Keywords: Knowledge, Attitudes, Nosocomial infections, Hospital Acquired Infections, Anaesthesia Practitioners, Operating theatres.

\section{Introduction}

Nosocomial infections also known as Healthcare Associated Infections or Hospital Acquired Infections (HAIs) place a significant economic burden on the healthcare system ${ }^{[1]}$. Infection control practices are important in minimizing healthcare associated infections. However, compliance with Universal and Standard Precautions has been reported in a number of studies to be low. Not many of these studies are from low and middle-income countries, which could have cultural, managerial, educational level, financial and environmental factors that may influence compliance differently from developed countries. World Health Organization (WHO) describes hospital acquired infection to be one of the major infectious diseases having huge economic impact worldwide ${ }^{[2]}$. These infections affect about 2 million people annually resulting in
$5 \%$ to $15 \%$ of them requiring hospitalization ${ }^{[3,4]}$. Anaesthesia practitioners are very paramount in nosocomial infection preventions. There are a lot of procedures anaesthetists undertake that breach the physiological barriers allowing for possible contamination of micro-organisms and consequent development of infection. An example of such procedures includes; tracheal intubation, venous access, neural blocks etc. Inadequate knowledge, negative attitude and non-adherence to standard or recommended practices can lead to micro-organism transmission from patient to patient, from anaesthetists to patients or from patients to anaesthetists. Observation of good hygiene, adequate cleaning of anaesthesia equipment, use of proper and clean protective clothing, adequate skin preparation of the patient etc. can reduce the risk of infection transmission. Healthcare acquired infections have been regarded as a public health problem globally 
and are on the increase despite efforts made by hospitals and their managements to control the rate of infection and contribute significantly to mortality and morbidity ${ }^{[5]}$. It is by nature that any micro-organism has the potential to cause infection in hospitalized patients but only a few including Staphylococci, Escherichia coli, Pseudomonas aeruginosa, Enterococci, fungi and to lesser extent, viruses and parasites are responsible for the majority of healthcare acquired infections ${ }^{[5]}$.

Patients are hospitalized with the aim to ensure that they recover from their illnesses but this is not always so. Sometimes, their health deteriorates and healthy people get infected in healthcare facilities. The occurrence and unwanted consequences of HAIs have been known for several decades and continue to escalate at an alarming rate ${ }^{[6]}$. HAIs constitute a serious global public health challenge, causing untold suffering to about 1.4 million people across the world at any given time ${ }^{[7]}$. They often increase costs of health care on both patients and health services ${ }^{[8]}$. Many HAIs are preventable as it has been shown that compliance with guidelines greatly reduces both the rate and number of infections ${ }^{[9]}$. Among the many factors responsible for continued increase of HAIs in hospitalized patients are: poor immune status of patients; extremes of age, use of medical procedures and/or invasive techniques/devices, emergence of drug-resistant bacteria and over- crowding in hospitals ${ }^{[10,11]}$. Poor infection control practices may also facilitate micro-organism transmission. Studies found that hand washing, education, personal hygiene, knowledge of risky practices, immunization, interaction with public health officials when illness occurs and interruption of faecal-oral spread $[11 ; 12 ; 13]$ are all essential for containment of HAIs. A good knowledge of health professional about HAIs will help reduce their prevalence among hospitalized patient and the health workers ${ }^{[14 ; 15]}$. HAIs has been reported to be high in sub-Saharan Africa and range between $21.2-35.6 \%{ }^{[16]}$ However, simple and effective control programmes together with effective training of healthcare workers will go a long way in reducing the endemic nature of nosocomial infections in sub Saharan Africa. A study conducted in Addis Ababa, Ethiopia to understand hospital infection control practices revealed that $90 \%$ of the healthcare workers had good knowledge of infection prevention practices ${ }^{[17]}$ However, there was knowledge-practice discrepancies. In a similar study in South West Ethiopia, to assess knowledge, attitude and practice towards infection control measures among General hospital staff resulted in a high knowledge but less practice ${ }^{[18]}$ The same knowledgepractice discrepancy was also identified in a study conducted in Uganda to describe infection control knowledge, attitudes and practices among healthcare workers indicated that almost all HCWs had knowledge on infection prevention ${ }^{[19]}$. A systematic review indicated high knowledge of majority of staff towards infection prevention and control but low knowledge-practice ${ }^{[20]}$. Many studies have reported varying levels of knowledge regarding infection control in HCWs, and the proportion of HCWs who were aware of these practices ranged from $16-75 \%$. A study of HCWs in Nepal reported that $16 \%$ of HCWs had knowledge of infection control ${ }^{[21]}$. A study in Jordan reported that $49.6 \%$ of HCWs had knowledge of infection control, whereas a study in India reported that $75.5 \%$ of staff nurses at a tertiary care hospital had knowledge of infection control ${ }^{[2 ; 23]}$. Attitude of healthcare staff mainly helps in reducing the rate of HAIs. There are approved and recommended attitudes that healthcare staff need to exhibit in the healthcare setting in other to prevent the spread of HAIs both at work and home. In a study that aims to establish baseline attitude towards infection control and compliance with specific Operating Department (OD) guidelines ${ }^{[24]}$, indicated that $87 \%$ of the staff at the OD always wash their hands after touching bodily fluids. It further indicated that $12 \%$ strictly adhered to the guideline (i.e. never recap used sharps and needles) however, $42 \%$ occasionally recap. Even though $68 \%$ of the respondents in the same study agreed with the policy of using face mask, the declared compliance rate is $86 \%$ and about $55 \%$ of the respondents complied with changing clothes on exit and re-entry into the OD.

Wide variations in compliance were recorded concerning individual infection control guidelines in various studies. Hand washing rate ranges from $27-86 \%$ with the average of $52 \%{ }^{[25]}$. It was reported by Gammon et al that, glove, gown and eye protection rates were on average $62 \%$. The same study reported that the average compliance rate of facemask use is $30 \%{ }^{[26]}$.

\section{Materials and Methods}

This research was conducted in the operating theatres of Tamale Teaching Hospital. Tamale Teaching Hospital is a referral hospital serving the three northern regions of Ghana. It is about 400 bed capacity facility. It also serves as the main teaching facility for the school of medicine and health sciences of university for development studies. It has about 10 operating theatres. A crosssectional study design was employed. A mixed-method approach was used. Both the questionnaire and observation methods were used to collect data at the TTH for the study. The study population consist of all anaesthetists at the TTH and the sample size was all the anaesthetists available during the period data collection. All anaesthetists were interviewed except three people who were on annual leave at the time of the study. The study duration was five months. It was done between the months of February 2017 to June 2017. The researcher took two days to train field officers on the research tool and how to manage data. The training programme was centred on methods of conducting oral interviews, taking detailed field notes during observation. A structured questionnaire was used to collect the data via face to face interview and observation. The first part of the questionnaire consisted of the demographic characteristics of the respondents such as age, sex, level of education and number of years of practice. Part two of the questionnaire captured respondent's knowledge on nosocomial infection prevention, attitude on hospital acquired infection prevention and hospital acquired infection prevention practices.

The questionnaire was administered using both a face-toface interview and observation. The advantage of using the face-toface interview was to eliminate non-response and incomplete data from the study and observation also help to see what the respondents actually do, although it is time consuming. To secure the consent of respondents, the study's purpose was explained to enable them to decide whether to participate or not. All anaesthesia personnel working at the TTH were included in the study. No anaesthesia personnel were excluded from the study except those on annual leave during the period of the data collection. Permission was sought from the research and ethical committee of the hospital through the department of anaesthesia, University for Development Studies. Consent was also sought from anaesthetists as well. Letters indicating the study's purpose and its intended start date was directly delivered to the managers of the facility. The managers subsequently reviewed and approved the study and indicated their readiness for the start of data collection. Statistical Package for the Social Science (SPSS)version 20 and descriptive statistics was used. Frequency tables and charts were used to display the necessary variables relevant to the study.

\section{Results}


Demographic characteristics of respondents are presented in Table 1. Below. Results indicate that majority of the respondents were male anaesthetists $(83.3 \%)$ against female anaesthetists of $16.7 \%$. More of the respondents were in the 30-34yrs age group (44.4\%) with $35-39 y$ rs forming $33.3 \%$ of the respondents. Majority of the respondents $(61.1 \%)$ holds an advanced level diploma certificate while $38.9 \%$ holds a bachelor's degree certificates. Table. 1 further depicts that half $(50 \%)$ of the respondents had working experiences of 0-4 years while $38.9 \%$ had 5-9years working experience.

Religious affiliation presented indicates that $55.6 \%$ of the respondents were Christians while $44.6 \%$ were Muslims.

Table 1: Demographic characteristics of respondents

\begin{tabular}{|l|c|c|}
\hline Demographic Characteristics & Frequency (n) & Percent (\%) \\
\hline Age & & \\
\hline 25-29yrs & 3 & 8.3 \\
30-34yrs & 16 & 44.4 \\
35-39yrs & 12 & 33.3 \\
40yrs + & 5 & 13.9 \\
\hline Gender & 30 & \\
\hline Male & 6 & 83.3 \\
Female & & 16.7 \\
\hline Educational Level & 22 & 61.1 \\
\hline Advanced Diploma & 14 & 38.9 \\
Bachelor Degree & & \\
\hline Working Experience & 18 & 50.0 \\
\hline 0-4yrs & 14 & 38.9 \\
5-9yrs & 4 & 11.1 \\
10yrs \& above & & \\
\hline Religious Affiliation & 20 & 55.6 \\
\hline Christian & 16 & 44.4 \\
Islamic & & \\
\hline
\end{tabular}

The availability of an infection prevention guideline presented in Table 2. Results from the table indicate that $83.3 \%$ of the respondents reported that recommended infection prevention guideline is available while $16.7 \%$ responded in the negative even though, all respondents (100\%) have knowledge on infection prevention guidelines. The source of information about infection prevention guidelines indicated that majority of the respondents (55.6\%) got the knowledge from attending training courses while $22.2 \%$ got it from own research and oral communication. $25 \%$ of the respondents reported that infection prevention and control (IPC) guidelines were readily available to see and read while $75 \%$ reported in the negative. As to whether the guideline affects their scope of work positively, $33.3 \%$ reported that the guideline does not affect their scope work anyway while $66.7 \%$ of the respondent's reported that the guideline affects their scope of work positively. Respondents' adherence to IPC guideline indicated that $72.2 \%$ adhere strictly to IPC practices while $27.8 \%$ does not adhere to it strictly.

Table 2: Knowledge about Infection prevention guideline

\begin{tabular}{|l|c|c|}
\hline Knowledge, Attitude \& Practice & $\begin{array}{c}\text { Frequency } \\
\text { (n) }\end{array}$ & $\begin{array}{c}\text { Percent } \\
(\%)\end{array}$ \\
\hline Knowledge on availability of IP & & \\
guideline & 30 & 83.3 \\
\hline Yes & 6 & 16.7 \\
No & & \\
\hline Source of Information & 8 & 22.2 \\
\hline Oral Communication & 8 & 22.2 \\
Own Research & 20 & 55.6 \\
Training Courses & & \\
\hline Guideline (protocol) readily & & \\
available & 9 & 25.0 \\
\hline Yes & 27 & 75.0 \\
No & & \\
\hline Guideline affect scope of work & 6 & 66.7 \\
\hline Yes & 3 & 33.3 \\
No & & \\
\hline Adhere strictly to IPC practices & 26 & 72.2 \\
\hline Yes & 10 & 27.8 \\
\hline No & & \\
\hline
\end{tabular}

Respondents' infection control practices in the operating theatre may be described as moderate. Results in Table 3 show that items that received the lowest mean scores were wearing of goggles (Mean $=0.58$; standard deviation $(\mathrm{SD})=0.77)$ and wearing of gowns $($ Mean $=0.83 ; \mathrm{SD}=0.94)$. Descriptive statistics have indicated that all $(100 \%)$ of the anaesthetists change their clothes when leaving the operating theatre to their various homes. The majority $(69.4 \%)$ of the respondents always change clothing's on exit and re-entry into the operating theatre. More than half of the respondents wear face masks $(63.9 \%)$ and wash hands before and after procedure. On Observation, majority of the respondents always and sometimes recap used needles, only $13.9 \%$ that do not recap used needles.

Table 3: Descriptive statistics of practices of infection control measures

\begin{tabular}{|l|c|c|c|c|c|}
\hline Infection prevention practices & Never (\%) & Sometimes (\%) & Always (\%) & Mean (1-3) & SD \\
\hline Hand washing before and after procedure & 0 & 36.1 & 63.9 & 2.64 & 0.487 \\
\hline Recap of used needles & 13.9 & 47.2 & 38.9 & 1.89 & 1.09 \\
\hline Wearing of face masks & 0 & 36.1 & 63.9 & 2.61 & 0.549 \\
\hline Wearing goggles & 58.3 & 41.7 & 0 & 0.58 & 0.77 \\
\hline Wearing of gloves & 0 & 25 & 75 & 2.75 & 0.439 \\
\hline Wearing gowns & 44.4 & 47.2 & 8.3 & 0.83 & 0.941 \\
\hline Change clothes on exit and re-entry & 5.6 & 25 & 69.4 & 2.44 & 0.939 \\
\hline Change clothes when leaving the theatre & 0 & 0 & 100 & 4 & 0 \\
\hline
\end{tabular}

PPE's are worn to protect one from getting in contact with dirt, blood, fluid or infection. During the tracheal intubation procedure, it was reported that all respondents (100\%) always wear gloves, $91.7 \%$ always wear face masks and $91.7 \%$ never wear goggles, and $88.9 \%$ never wear sterile gowns (Table 4). However, all the protective clothing's are needed during the procedure.

Table 4: Personal Protective Equipment (PPE) usage by anaesthesia practitioners during Tracheal intubation

\begin{tabular}{|l|c|c|}
\hline Procedure & Frequency $(\mathbf{n})$ & Percent (\%) \\
\hline Tracheal intubation & & \\
\hline Face Mask & & \\
\hline Always & 33 & 91.7 \\
Sometimes & 2 & 5.6 \\
Never & 1 & 2.8 \\
\hline
\end{tabular}




\begin{tabular}{|l|c|c|}
\hline Gloves & & \\
\hline Always & 36 & 100 \\
\hline Sterile Gown & & \\
\hline Always & 1 & 2.8 \\
Sometimes & 3 & 8.3 \\
Never & 32 & 88.9 \\
\hline Goggles & 1 & \\
\hline Always & 2 & 2.8 \\
Sometimes & 33 & 5.6 \\
Never & & 91.7 \\
\hline
\end{tabular}

PPE's worn during extubation are presented in Table 5 below. Results indicate that $91.7 \%$ of the respondents wear face masks always and $100 \%$ always wear gloves. However, $97.2 \%$ never wear goggles during the procedure while $86.1 \%$ never wear sterile gowns.

Table 5: PPE usage by Anaesthesia practitioners during extubation

\begin{tabular}{|l|c|c|}
\hline Procedure & Frequency (n) & Percent (\%) \\
\hline Extubation & & \\
\hline Mask & & \\
\hline Always & 33 & 91.7 \\
Sometimes & 3 & 8.3 \\
\hline Gloves & & \\
\hline Always & 36 & 100 \\
\hline Sterile Gown & & \\
\hline Always & 2 & 5.6 \\
Sometimes & 3 & 8.3 \\
Never & 31 & 86.1 \\
\hline Goggles & & \\
\hline Sometimes & 1 & 2.8 \\
Never & 35 & 97.2 \\
\hline
\end{tabular}

Results from Table. 6 presented PPE's worn during regional block, indicated that $88.9 \%$ of anaesthetists always wear face masks and $100 \%$ wear gloves. Yet, $80.6 \%$ and $69.4 \%$ never wear goggles and sterile gowns respectively.

Table 6: PPE usage by anaesthesia practitioners during regional block

\begin{tabular}{|l|c|c|}
\hline Procedure & Frequency (n) & Percent (\%) \\
\hline Regional block & & \\
\hline Mask & & \\
\hline Always & 32 & 88.9 \\
Sometimes & 3 & 8.3 \\
Never & 1 & 2.8 \\
\hline Gloves & & \\
\hline Always & 36 & 100 \\
\hline Sterile gown & & \\
\hline Always & 1 & 2.8 \\
Sometimes & 10 & 27.8 \\
Never & 25 & 69.4 \\
\hline Goggles & 7 & \\
\hline Sometimes & 29 & 19.4 \\
Never & \multicolumn{2}{|}{} \\
\hline
\end{tabular}

Table 7 presents PPE's worn during peripheral block. Results indicate that $100 \%$ always wear gloves and $52.8 \%$ always wear face masks during the procedure. Hitherto, $86.1 \%$ and $83.3 \%$ never wear goggles and sterile gowns respectively.
Table 7: PPE usage by anaesthesia practitioners during peripheral block

\begin{tabular}{|l|c|c|}
\hline Procedure & Frequency (n) & Percent (\%) \\
\hline Peripheral & & \\
\hline Mask & & \\
\hline Always & 19 & 52.8 \\
Sometimes & 13 & 36.1 \\
Never & 4 & 11.1 \\
\hline Gloves & & \\
\hline Always & 36 & 100 \\
\hline Sterile gown & & \\
\hline Always & 1 & 2.8 \\
Sometimes & 5 & 13.9 \\
Never & 30 & 83.3 \\
\hline Goggles & & \\
\hline Always & 1 & 2.8 \\
Sometimes & 4 & 86.1 \\
Never & 31 & \\
\hline
\end{tabular}

Results from Table 8 which presents PPE's worn during vascular access indicate that $100 \%$ of respondents always wear gloves. However, $97.2 \%, 91.7 \%$ and $63.9 \%$ never wear goggles, sterile gowns and face masks respectively during the procedure.

Table 8: PPE usage by anaesthesia practitioners during vascular access

\begin{tabular}{|l|c|c|}
\hline Procedure & Frequency (n) & Percent (\%) \\
\hline Vascular Access & & \\
\hline Mask & & \\
\hline Always & 4 & 11.1 \\
Sometimes & 9 & 25 \\
Never & 23 & 63.9 \\
\hline Gloves & & \\
\hline Always & 36 & 100 \\
\hline Sterile gown & & \\
\hline Sometimes & 3 & 8.3 \\
Never & 33 & 91.7 \\
\hline Goggles & 1 & \\
\hline Sometimes & 35 & 2.8 \\
Never & & 97.2 \\
\hline
\end{tabular}

Distribution of hand hygiene practice is represented in Figure 1and Figure 2 below. Results indicate that only $31 \%$ of the respondents reported that alcohol hand gel is readily available at the theatre while $69 \%$ reported that it is not always available. From the analysis on availability of water and soap for hand washing indicated that $78 \%$ reported water and soap is always available for hand washing while $22 \%$ reported otherwise.

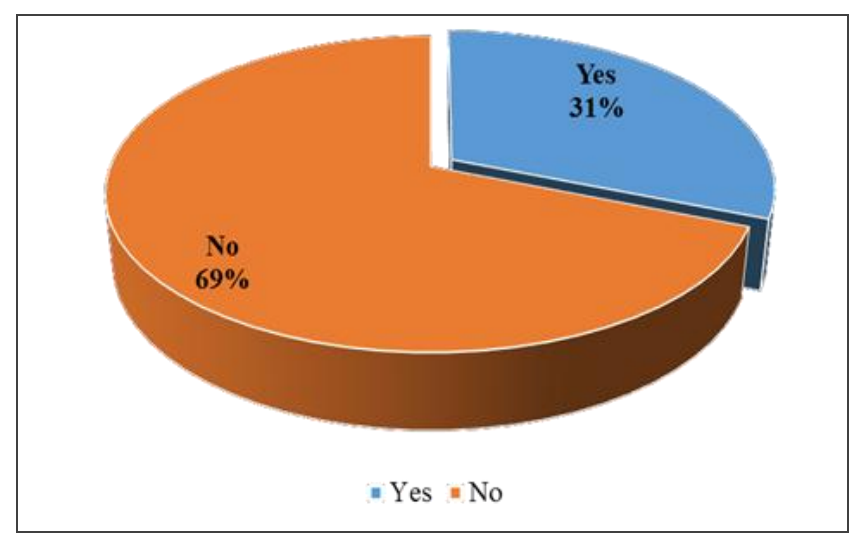

Figure 1: Availability of alcohol hand gel in the operating theatres 


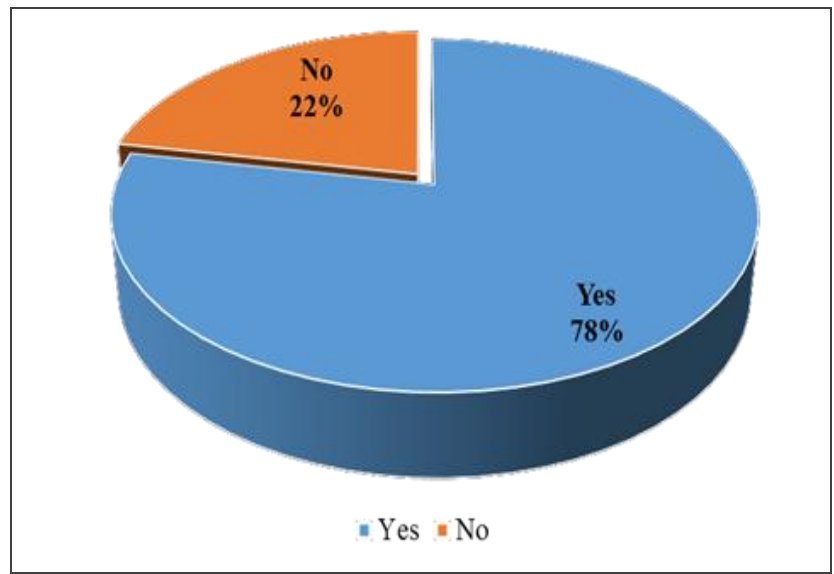

Figure 2: Availability of Soap and water in the operating theatres

Situations of hand washing is presented in the Table 9. The results from the analysis indicated that at the beginning of the days' work, $58.3 \%$ of respondents sometimes wash their hands, $27.8 \%$ always wash their hands at the beginning of the day and $13.9 \%$ never wash their hands at the beginning of the day but observation revealed that none $(0)$ wash their hands always at the beginning of the day and majority (75\%) sometimes wash their hands at the beginning of the day. In between cases, it was reported that $58.3 \%$ of respondents always wash their hands, $33.3 \%$ sometimes wash their hands in between cases but $8.3 \%$ never wash their hands in between cases whereas observation indicated that only $22.2 \%$ always wash their hands in between cases. After coming into contact with patient, only one (1) respondents representing $(2.8 \%)$ never wash hands but $63.9 \%$ always wash their hands while $33.3 \%$ sometimes wash hands after coming into contact with patient and observation indicated that majority $(80.6 \%)$ sometimes wash their hands. Before and after inducing anaesthesia, $44.4 \%$ always wash their hands but $47.2 \%$ sometimes wash their hands and $8.3 \%$ never wash their hands before and after inducing anaesthesia whereas on observation, only $13.9 \%$ always wash their hands but $77.8 \%$ sometimes wash their hands before and after inducing anaesthesia. Before and after regional block procedure, it was reported from the analysis that $75 \%$ of respondents always wash their hands, $22.2 \%$ sometimes wash their hands and $2.8 \%$ never wash their hands whereas observation revealed that only $33.3 \%$ always wash their hands and $61.1 \%$ sometimes wash their hands before and after regional block. Before and after other procedures, it was reported that $69.4 \%, 52.8 \%$ and $47.2 \%$ of respondents always wash their hands before and after peripheral block, vascular access and before and after removing gloves but it was observed that only $11.1 \%$ always wash their hands and $86.1 \%$ sometimes wash their hands before and after peripheral block. $88.9 \%$ and $77.8 \%$ sometimes wash their hands before and after vascular access and before wearing and after removing gloves respectively. However, $2.8 \%$ never wash their hands before and after peripheral block. Also, $2.8 \%$ and $8.3 \%$, never wash their hands before and after removing of gloves respectively.

Table 9: Situations of Hand Washing by anaesthesia practitioners (Self-reported and observed)

\begin{tabular}{|c|c|c|c|c|}
\hline Situations of hand washing & $\begin{array}{c}\text { Self-Reported (Response) } \\
\text { Frequency(n) }\end{array}$ & Percent (\%) & $\begin{array}{c}\text { Observed } \\
\text { Frequency(n) }\end{array}$ & Percent (\%) \\
\hline \multicolumn{5}{|l|}{ Beginning the day } \\
\hline Never & 5 & 13.9 & 9 & 25.0 \\
\hline Sometimes & 21 & 58.3 & 27 & 75.0 \\
\hline Always & 10 & 27.8 & 0 & 0.0 \\
\hline \multicolumn{5}{|l|}{ In between cases } \\
\hline Never & 3 & 8.3 & 8 & 22.2 \\
\hline Sometimes & 12 & 33.3 & 20 & 55.6 \\
\hline Always & 21 & 58.3 & 8 & 22.2 \\
\hline \multicolumn{5}{|c|}{ After coming into contact with a patient } \\
\hline Never & 1 & 2.8 & 3 & 8.3 \\
\hline Sometimes & 12 & 33.3 & 29 & 80.6 \\
\hline Always & 23 & 63.9 & 4 & 11.1 \\
\hline \multicolumn{5}{|c|}{ Before and after inducing anaesthesia } \\
\hline Never & 3 & 8.3 & 3 & 8.3 \\
\hline Sometimes & 17 & 47.2 & 28 & 77.8 \\
\hline Always & 16 & 44.4 & 5 & 13.9 \\
\hline \multicolumn{5}{|c|}{ Before and after regional block } \\
\hline Never & 1 & 2.8 & 2 & 5.6 \\
\hline Sometimes & 8 & 22.2 & 22 & 61.1 \\
\hline Always & 27 & 75.0 & 12 & 33.3 \\
\hline \multicolumn{5}{|c|}{ Before and after peripheral blocks } \\
\hline Never & 1 & 2.8 & 1 & 2.8 \\
\hline Sometimes & 10 & 27.8 & 31 & 86.1 \\
\hline Always & 25 & 69.4 & 4 & 11.1 \\
\hline \multicolumn{5}{|c|}{ Before and after vascular access } \\
\hline Never & 1 & 2.8 & 3 & 8.3 \\
\hline Sometimes & 16 & 44.4 & 32 & 88.9 \\
\hline Always & 19 & 52.8 & 1 & 2.8 \\
\hline \multicolumn{5}{|c|}{ Before wearing and after removing gloves } \\
\hline Never & 3 & 8.3 & 8 & 22.2 \\
\hline Sometimes & 16 & 44.5 & 28 & 77.8 \\
\hline Always & 17 & 47.2 & 0 & 0.0 \\
\hline
\end{tabular}


Cleaning of anaesthesia equipment is important in the infection prevention and control practice. It was observed that $66.7 \%$ of the respondents always clean anaesthesia equipment while $30.6 \%$ clean it sometimes and 2.8\% never clean anaesthesia equipment (Figure 3). But $91.7 \%$ of the respondents use $0.5 \%$ chlorine or spirit to clean the equipment while 2.8 use ordinary water to clean and
$5.6 \%$ did not know what is used to clean the equipment. Anaesthesia equipment cleaned is presented in (Figure 4) and results indicate that Laryngoscope and Guedell airways are always $100 \%$ kept clean. However, $94.4 \%$ of the anaesthetists clean Supraglottic devices always while $75 \%$ clean Suction tubes always and $66.7 \%$ always clean Magill forceps.

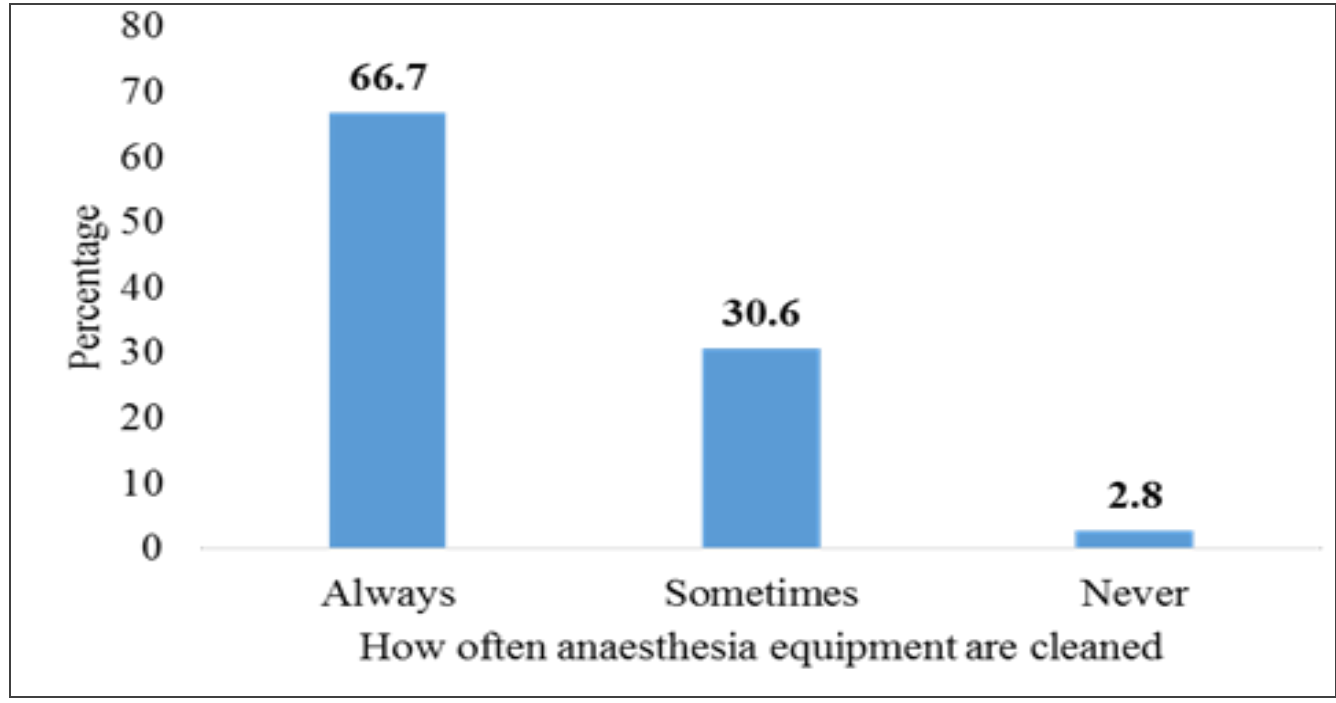

Figure 3: Frequency of Cleaning of anaesthesia equipment by anaesthesia practitioners

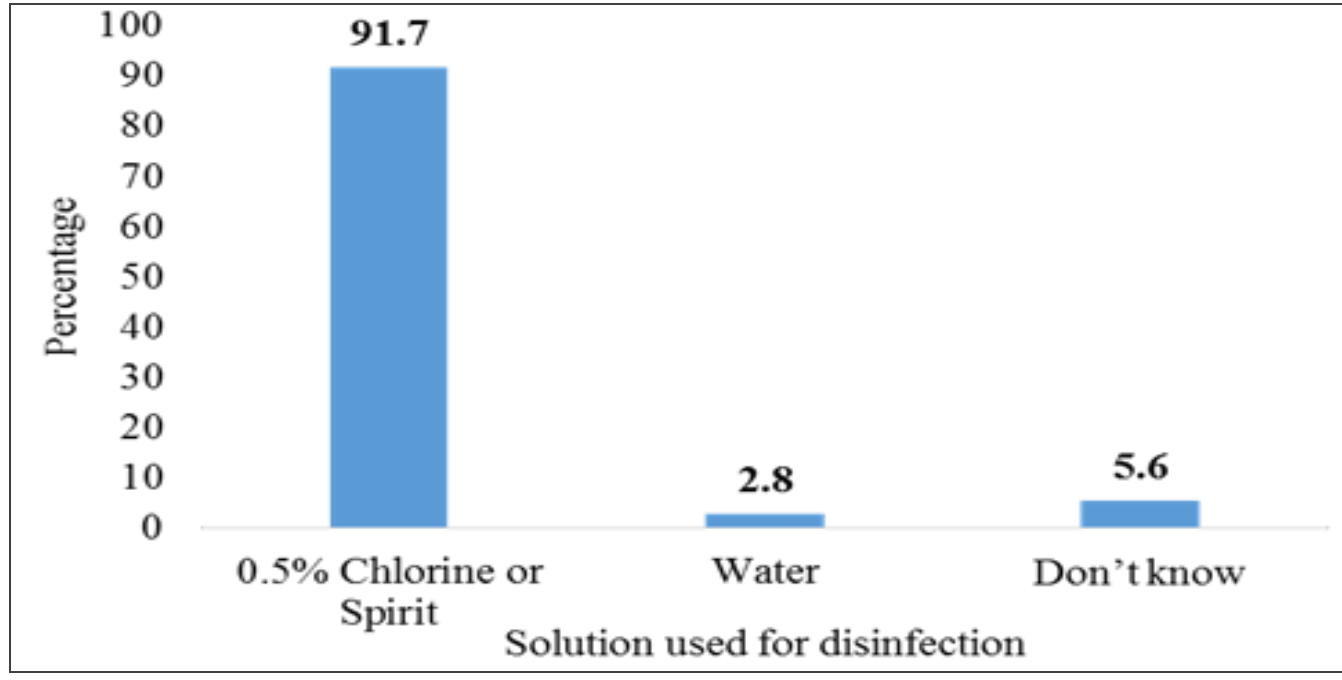

Figure.4: Types of substances used in cleaning anaesthesia equipment

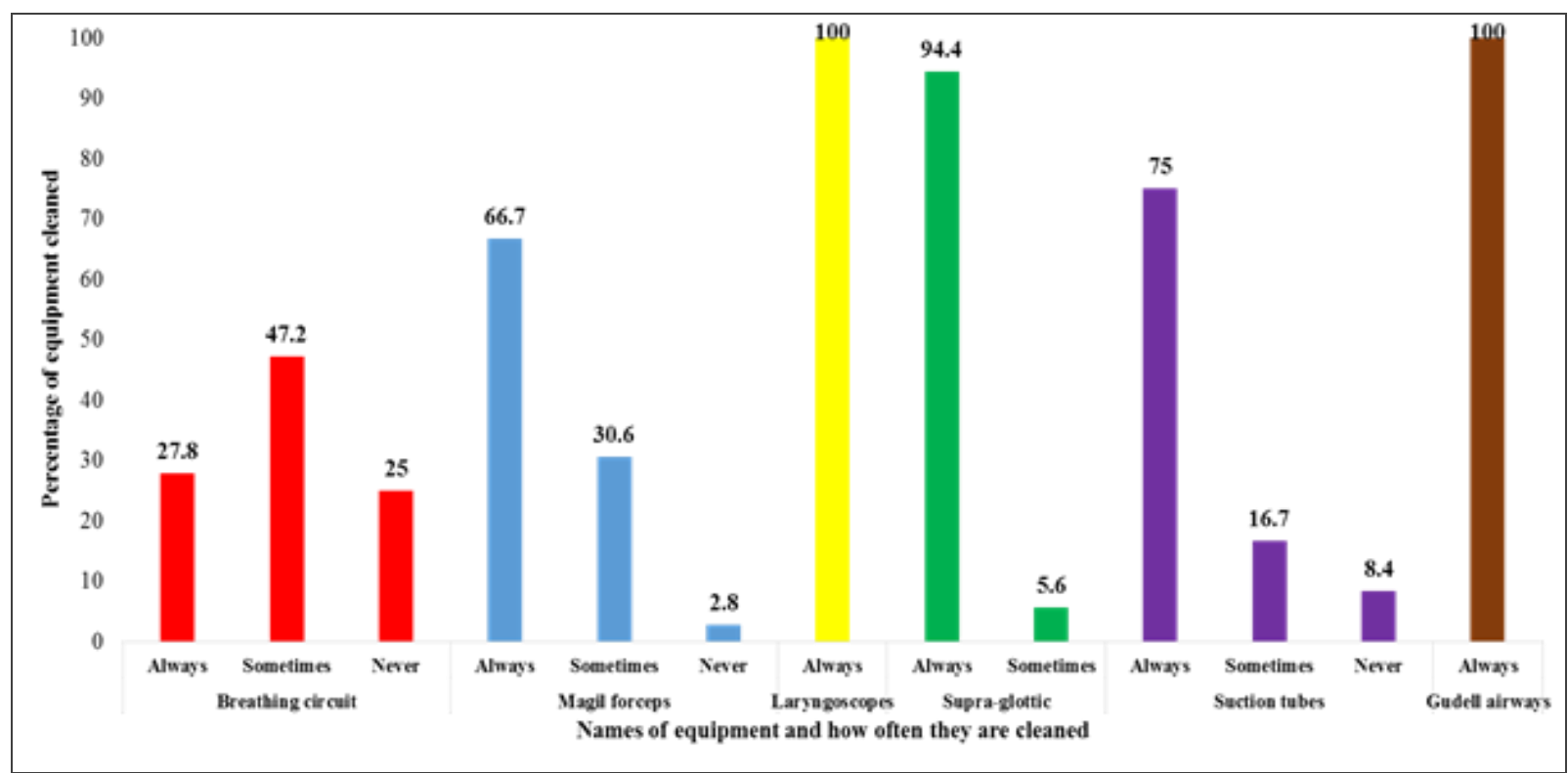

Figure 5: Types of Anaesthesia equipment cleaned by anaesthesia practitioners 
The Laryngoscopes were the most cleaned always (100\%) by anaesthesia practitioners according to the results depicted in Figure. 5

An overwhelming number (89\%) of respondents change breathing circuit after infected or high- risk patient is attended to (Figure 6).

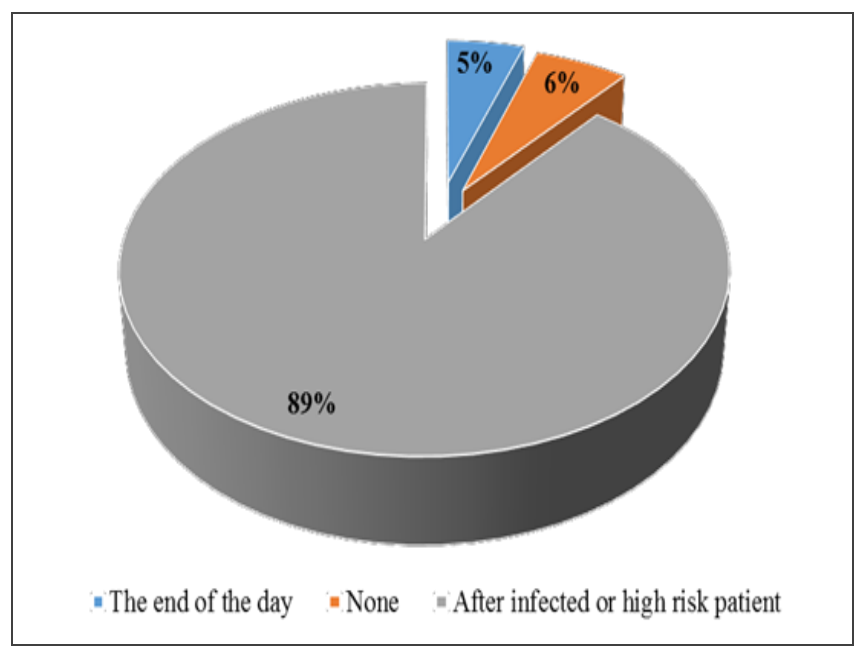

Figure 6: Changing of breathing circuit by anaesthesia practitioners

Drugs use and kept for re-use information is contained in Table 10 and results indicate majority of the drugs when opened, should be kept for re-use within 24 hours an evidenced by $91.7 \%$ of respondents reported for Propofol, 61.1\% of respondents reported for Atropine,55.6\% of respondents reported for Thiopental and $52.8 \%$ of respondents reporting for Succinylcholine.

Table 10: Safe injection practices and drug use by anaesthesia practitioners

\begin{tabular}{|l|c|c|}
\hline Duration & Frequency (n) & Percent (\%) \\
\hline Propofol & & \\
\hline Within 24 hrs & 33 & 91.7 \\
Don't know & 3 & 8.3 \\
\hline Thiopental & & \\
\hline Within 24 hrs & 20 & 55.6 \\
After 48 hrs & 12 & 33.3 \\
Don't know & 4 & 11.1 \\
\hline Atropine & & \\
\hline Within 24 hrs & 22 & 61.1 \\
After 48 hrs & 11 & 30.6 \\
Don't know & 3 & 8.3 \\
\hline Succinylcholine & & \\
\hline Within 24 hrs & 19 & 52.8 \\
After 48 hrs & 12 & 33.3 \\
Don't know & 5 & 13.9 \\
\hline
\end{tabular}

Majority of the respondents (89\%) use new syringes to draw fraction of drug for use for a patient while $11 \%$ does not use new syringe (Figure.7). However, all (100\%) of the respondents discard used syringes and needles in a sharp container.

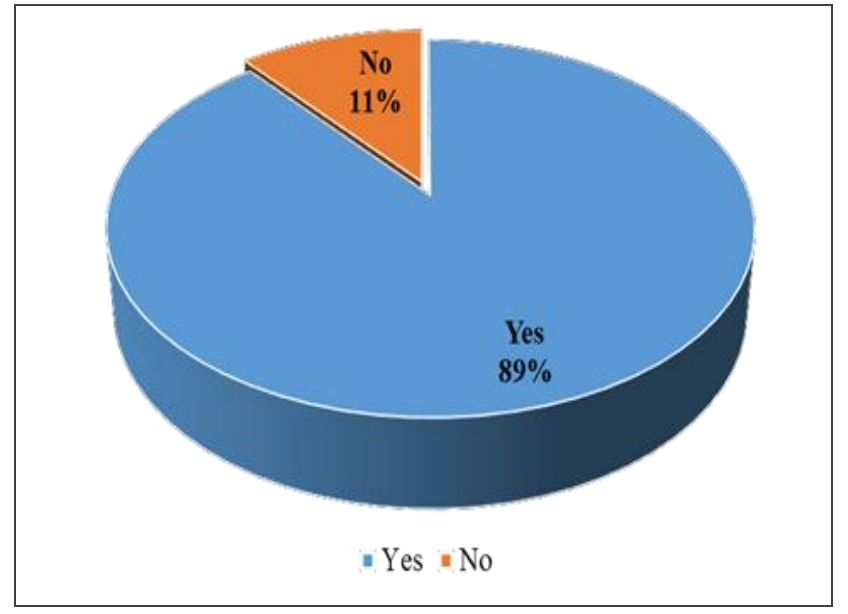

Figure 7: Use of new syringe to withdraw fraction of drug by anaesthesia practitioners

\section{Discussions}

Demographic characteristics of respondents indicate that majority of the respondents were male anaesthetists $(83.3 \%)$ against female anaesthetists of $16.7 \%$. More of the respondents $(44.4 \%)$ were in the age group of 30-34years while 35-39years forms $33.3 \%$. Majority of the respondents $(61.1 \%)$ holds an advanced level diploma certificate while $38.9 \%$ holds a bachelor's degree certificates. Furthermore, half $(50 \%)$ of the respondents had working experiences of $0-4$ years while $38.9 \%$ had 5-9years working experience. Religious affiliation presented indicates that $55.6 \%$ of the respondents were Christians while $44.4 \%$ were Muslims.

Results from the study indicated that $83.3 \%$ of the respondents reported that a recommended infection prevention guideline is available while $16.7 \%$ responded in the negative. However, all respondents $100 \%$ have knowledge on infection prevention and control guidelines. The source of knowledge on infection prevention guidelines indicated that majority of the respondents $55.6 \%$ got the knowledge from attending training courses while $22.2 \%$ got it from their own research and oral communication. Infection prevention and control (IPC) guidelines(protocol) were reported to be readily available by $25 \%$ of the respondents whilst $75 \%$ reported in the negative. As to whether the guideline affects their scope of work positively, it was realized that the guideline affects $66.7 \%$ of the respondent's scope of work positively while $33.3 \%$ of the respondent's scope of work was not affected by the guideline. Respondents' adherence to IPC guideline indicated that $72.2 \%$ adhere strictly to IPC practices while $27.8 \%$ do not adhere to it strictly. This therefore means that anaesthetists at TTH have adequate knowledge on infection prevention control.

These findings corroborated with Adamasuet al, (2013) in Ethiopia and Yakob, Lamoro \& Henok, (2015) which assessed infection control practices among hospital staff. This study also agreed with Sethi et al, (2012) in Uganda in which almost all healthcare workers had knowledge in infection prevention and again supported by Gould et al, (2010) in a systematic review which indicated a high knowledge of infection prevention among healthcare workers. However, the findings of this study were contrary to Paudyal et al, (2008) in Nepal which reported knowledge of infection prevention at $16 \%$ and Taneja et al, (2009) and Darawad\& Al-Hussami, (2012) who reported $75.5 \%$ knowledge in a tertiary care hospital. 
Findings from the study on attitude and practice are group into the following areas; personal hygiene, personal protective equipment, cleaning of anaesthesia equipment, skin preparations and safe injection practices.

Results on hand hygiene indicated that only $30 \%$ of the respondents reported alcohol hand gel is readily available at the operating theatres while $69 \%$ reported in negative. $78 \%$ of the respondents indicated that, water and soap are always available in the theatre for hand washing and $22 \%$ of respondents reported otherwise. $36.1 \%$ sometimes perform hand washing before and after each procedure while $63.9 \%$ always wash their hands before and after each procedure. On the other hand, only one respondent (2.8\%) failed to wash hand when come into contact with patient, the rest $33.3 \%$ and $63.9 \%$ of respondents wash their hands sometimes and always respectively. Furthermore, 27 respondents reported they always wash their hands before and after regional block. But on observation, only 12 (33.3\%) always wash their hands before and after regional block.

Findings on personal protective clothing's in regards to attitude and practice vary. The result from the study indicated that all $(100 \%)$ of the anaesthetists change their clothing when leaving the operating theatre to their various homes. Again, majority $(69.4 \%)$ of the respondents always change clothing on exit and reentry into the operating theatre. Wearing of gloves had higher positive attitude and practice with a score of $100 \%$ followed by face mask which scored $36.1 \%$ and $63.9 \%$ respectively for sometimes and always. Wearing of goggles and sterile gowns had lowest score towards the attitude and practice on HAIs prevention control. Ranging from $69.4 \%$ to $91.7 \%$ of respondents do not wear sterile gowns when performing procedures such as extubation, intubation, regional anaesthesia, peripheral blocks etc. while ranging from $80.6 \%$ to $97.2 \%$ of respondents do not wear goggles when performing same procedures.

Cleaning of anaesthesia equipment is very important in infection prevention and control. From the study, it was noted that $66.7 \%$ of respondents' always clean anaesthesia equipment after procedure while $30.6 \%$ respondents sometimes clean but $2.8 \%$ respondents do not clean at all after the procedure. $91.7 \%$ respondents use $0.5 \%$ chlorine or spirit for cleaning of the anaesthesia equipment while $2.8 \%$ use only water for cleaning. $5.6 \%$ respondents do not know what is used in cleaning the anaesthesia equipment.

Attitude and practice on infection prevention and control among anaesthetists on skin preparation before a procedure according to the finding of this study was optimal. All 36(100\%) respondents clean the skin twice with antiseptic such as savlon and spirit before undertaking procedures such regional and peripheral blocks.

Respondent's attitude and practice towards safe injection practices followed the standard on one side while the other did not follow the standard. $100 \%$ of the respondents discard used syringes and needles in sharp container whilst as low as only $13.9 \%$ do not recap used needles which is the standard.

This result concluded that infection control practices in the operating theatres at TTH were moderate. This can also be described as suboptimal levels of compliance with standard of infection control guideline.

This finding complied with the findings of general studies on HCW compliance with infection control policies in developed countries (Stein et al, 2003; Gammon et al, 2008; Gershon et al, 1996). Again, the findings from the study support the previous reports that HCWs are selective in their adherence to individual guidelines. Variations with individual infection control guidelines have been reported in many studies. For hand washing, compliance ranges from $27-86 \%$, with a mean of $52 \%$ (Pittetet al, 1999). Regarding PPE, Gammon et al. reported that gloves, gown and goggles compliance rates were, on average 62\% (range 11-98\%), $57 \%$ (range $8-93 \%$ ) and 38 (range 0-92\%) respectively. Gammon et al also found the mean compliance rate for face mask to be 30 $(4-55 \%)$.

\section{Study Limitations}

- The observational aspect of the data collection was time consuming and also the duration of the study is limited.

- Limited resources especially financial constraints were a very big challenge to the researchers making it impossible for the authors to extend the study to other facilities.

- Anaesthesia staffs' co-operation during the data collection was challenge due to their tight work schedules.

\section{Conclusions}

In conclusion, this study demonstrated that anaesthetists at the TTH have reported sub-optimal levels of compliance i.e. attitude and practice with selective infection control. The study further demonstrated that discrepancies exist between anaesthetists' attitude towards a guideline as well as their actual practice. This suggests that multiple factors play a role in determining an anaesthetist's behaviour which in turn might explain why compliance to infection control precautions are internationally suboptimal.

\section{Recommendations}

- Infection control guidelines should be made available at the theatre so that anaesthetists and other health care workers in the department can have access to it and thereby comply with appropriate measures.

- Regular in-service training on IPC for anaesthesia staffs to update them on current infection prevention and control practices and the benefits of complying.

- Regular supply of $0.5 \%$ Chlorine solution and antiseptic to enable recommended cleaning of Anaesthesia equipment.

- $\quad$ Supportive supervision should frequently be carried out in the department to monitor the infection control practices of anaesthetists.

\section{Data availability statement}

The mixed quality and quantitative data used to formulate the conclusions of this study are all included in this manuscript

\section{Source of Funding}

The activities of this research were fully funded from monies contributed by the researchers. The authors did not receive any financial support from any institution or individual

\section{Conflict of interest}

We the authors of this research have no form of conflict of interest to declare 


\section{References}

[1] Plowman, R., Graves, N., Griffin, M. A. S., Roberts, J. A., Swan, A. V., Cookson, B., \& Taylor, L... The rate and cost of hospital-acquired infections occurring in patients admitted to selected specialties of a district general hospital in England and the national burden imposed. Journal of hospital infection 2001, 47(3), 198209.

[2] WHO. (1981). Surveillance, Control and Prevention of hospital Acquired (Nosocomial) Infection Report of Advisory group/NIC.

[3] Bock Avalos S, K. O. (2010 June). Nosocomial Infection, Nursing. URL; http: // find articles.com//articles/miqa3689/is200411/ai n9471334.

[4] Anusha, S., Vijaya, L. D., Pallavi, K., Manna, P. K., Mohanta, G. P., \&Manavalan, R... An epidemiological study of surgical wound infections in a surgical unit of tertiary care teaching hospital. Indian Journal of Pharmacy Practice 2010, 3(4).

[5] Mbim, E. N., Mboto, C. I., \&Agbo, B. E... A Review of Nosocomial Infections in Sub- Saharan Africa. British Microbiology Research Journal 2016, 15, 1-11.

[6] Collins, A. S. (2008). Preventing health care-associated infections.

[7] Pittet, D., \& Donaldson, L. Challenging the world: patient safety and health care-associated infection. International Journal for Quality in Health Care 2006, 18(1), 4-8.

[8] Kim, T., Oh, P. I., \& Simor, A.E. The economic impact of methicillin-resistant Staphylococcus aureus in Canadian hospitals. Infection Control \& Hospital Epidemiology 2001, 22(02), 99-104.

[9] Pittet, D., Hugonnet, S., Harbarth, S., Mourouga, P., Sauvan, V., Touveneau, S., \& Perneger, T. V... Effectiveness of a hospital-wide programme to improve compliance with hand hygiene. The Lance t 2000, 356(9238), 1307-1312.

[10] VINCat.Generalitat de Catalunya. (2010) OMS Nosocomial Infection; http://vincat.genlcat.cat/en/professional_sanitaris/la_infec cio_nosocomial/ Accessed on $12^{\text {th }}$ January, 2017

[11] Saka, M.J., Saka, A. O., \& Adebara, V. O. Prevention of Nosocomial Infections in the new born: The practice of private health facilities in rural communities of Nigeria. International Infection Diseases 2011, 1,9.

[12] Jamison, D. T., Breman, J. G., Measham, A. R., Alleyne, G., Claeson, M., Evans, D. B., \& Musgrove, P. (2006). Cost-effective strategies for the excess burden of disease in developing countries.

[13] Pellizzer, G., Mantoan, P., Timillero, L., Allegranzi, B., Fedeli, U.Schievano, E., \& Spolaore, P..Prevalence and risk factors for nosocomial infections in hospitals of theVeneto region, north-eastern Italy. Infection 2008, 36(2), 112-119.

[14] Ocran, I., \& Tagoe, D. N. A... Knowledge and attitude of healthcare workers and patients on healthcare associated infections in a regional hospital in Ghana. Asian Pacific Journal of Tropical Disease 2014, 4(2), 135-139.

[15] Amoran, O. E., \&Onwube, O. O... Infection control and practice of standard precautions among healthcare workers in northern Nigeria. Journal of global infectious diseases 2013, 5(4), 156.

[16] Mbim, E. N., Mboto, C. I., \&Agbo, B. E... A Review of Nosocomial Infections in Sub- Saharan Africa. British Microbiology Research Journal 2016, 15, 1-11.

[17] Adamasu, T., Stenehjem, E. A., Margoles, L., Kacha, E., Blumberg, H. M., \&Kempker, R.R... Infection control knowledge, attitudes, and practices among healthcare workers in Addis Ababa, Ethiopia. Infection Control \& Hospital Epidemiology 2013, 34(12), 1289-1296.

[18] Yakob, E., Lamoro, T., \&Henok, A... Knowledge, attitudes and practice towards infection control measures among Mizan-Aman general hospital workers, South West Ethopia. J Community Med Health Educ 2015, 5(5), 1-8.

[19] Sethi, A. K., Acher, C. W., Kirenga, B., Mead, S., Donskey, C. J., \&Katamba, A... Infection control knowledge, attitudes, and practices among healthcare workers at Mulago Hospital, Kampala, Uganda. Infection Control \& Hospital Epidemiology 2012, 33(09), 917923.

[20] Gould, D. J., Moralejo, D., Drey, N., \&Chudleigh, J. H. (2010). Interventions to improve hand hygiene compliance in patient care. The Cochrane Library.

[21] Paudyal, P., Simkhada, P., \& Bruce, J... Infection control knowledge, attitude, and practice among Nepalese health care workers. American journal of infection control 2008, 36(8), 595-597.

[22] Darawad, M. W., \& Al-Hussami, M... Jordanian nursing students' knowledge of, attitudes towards, and compliance with infection control precautions. Nurse education today 2013, 33(6), 580-583.

[23] Taneja, N., Chatterjee, S. S., Singh, M., Singh, S., \& Sharma, M. (2010). Pediatric urinary tract infections in a tertiary care center from north India.

[24] McGaw, C. D., Tennant, I., Harding, H. E., Cawich, S. O., Crandon, I. W., \& Walters, C. A... Healthcare workers' attitudes to and compliance with infection control guidelines in the operating department at the university hospital of the West Indies, Jamaica. International Journal of Infection Control 2012, $8(3)$.

[25] Pittet D, Mourouga P, Perneger TV, and the Members of the Infection Control Program. Compliance with handwashing in a teaching hospital. Ann Intern Med 1999; 130: 126-130

[26] Gammon, J., Morgan-Samuel, H., \& Gould, D... A review of the evidence for suboptimal compliance of healthcare practitioners to standard/universal infection control precautions. Journal of clinical nursing 2008, 17(2), 157-167. 